\title{
Prospective Study of the New Diffractive Bifocal Intraocular Lens
}

\author{
S. P. B. PERCIVAL \\ Scarborough
}

\begin{abstract}
Summary
The visual results of 55 bifocal lens implantations are compared with 55 matched PMMA monofocal implantations. $84 \%$ of the eyes with bifocal implants compared with $20 \%$ of the eyes with monofocal implants could read $N 8$ or better with the distance correction $(p<0.001) .52 \%$ of $+3.5 \mathrm{D}$ bifocal eyes could read N5 with the distance correction. The mean reading addition for a near point of $25 \mathrm{~cm}$ was $0.3 \mathrm{D}$ in the bifocal group and 2.2D in the monofocal group. $20 \%$ of eyes with bifocal implants could not be corrected to $\mathrm{N} 5$ at a comfortable distance $(p=0.005)$. It was found that the clear advantages of this bifocal lens must be countered in a minority by a loss in quality of vision particularly for reading.
\end{abstract}

There are several suggested ways of designing a bifocal lens. ${ }^{1}$ The first is a two zone refractive system in which the centre $2 \mathrm{~mm}$ of the lens focuses for near and the peripheral part focuses for distance. ${ }^{2}$ The two zones will have equal areas if the pupil is $2.8 \mathrm{~mm}$ diameter. But if the pupil is much smaller or larger or the lens is decentered, the area ratio changes and the bifocal function will diminish. A second design could counteract this problem by having multiple zones in which the even numbered ones focus for near and the odd numbered ones focus for distance. However, the zone widths would have to be so narrow that the simplicity of refractive optics would be lost because of diffractive aberration.

The design under current investigation (Fig. 1) makes use of diffraction optics using multiple zones with stepwise discontinuities on the posterior surface whereby light is diffracted towards two foci. Diffraction can be interpreted as a spreading of wave fronts. After encountering discontinuities, if the waves are in phase they interfere constructively, if they are out of phase, they interfere destructively. The design of the lens allows approximately $41 \%$ of light to be in phase and focused for near, $41 \%$ to be in phase and focused for distance, and $18 \%$ lost. ${ }^{1}$ Every zone has the same two foci and the bifocal function is therefore independent of pupil size and lens centration. Although of bifocal design the lens is often termed multifocal because many patients have been found to have an excellent depth of focus.

This paper is concerned with the assessment of comparative results with a monofocal lens and in particular with the quality of vision.

\section{Material and Methods}

Each of 55 patients with a bifocal lens in one eye was matched for age and sex with a patient with a PMMA monofocal lens. All lenses had angulated flexible loops inserted into the bag of the lens capsule with visual verification of this before the close of surgery. Intercapsular 


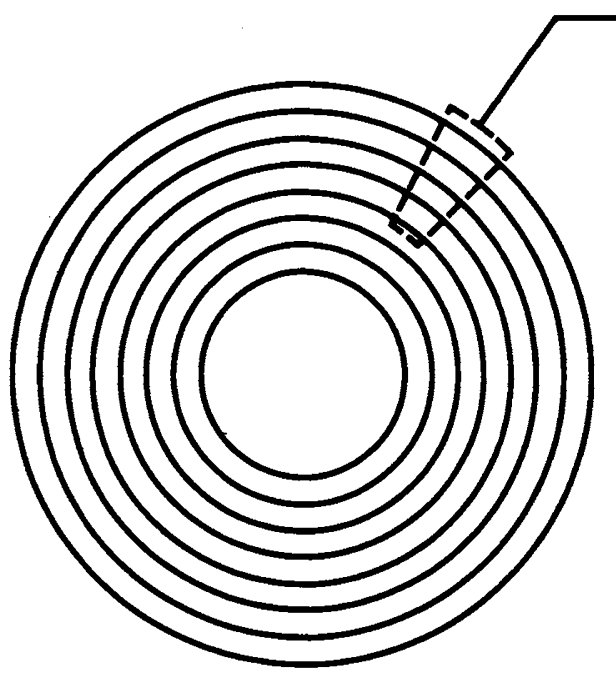

Fig. 1. Diagram of $3 M$ diffractive bifocal lens.

surgery was performed throughout. All controls operated on during the previous year were seeing $20 / 30$ or better six months after surgery.

Six months after surgery an assessment of reading ability was made by determining the smallest print seen with the distance correction and the reading addition necessary for a near point of $25 \mathrm{~cm}$. Each patient was provided with a formal questionnaire to determine the visual satisfaction, experience in different illuminations and presence of symptoms. Best corrected acuity was recorded at six metres for distance and between 35 and $25 \mathrm{~cm}$ for near.

The first five bifocal lenses implanted had à focus difference of $2.5 \mathrm{D}$ (equivalent to a spectacle reading addition of $1.7 \mathrm{D})$, the next fifty had a difference of 3.5D (equivalent to a reading addition of 2.3D), 47. were matched with other patients, eight had fellow eyes with monofocal lenses which were used as their controls. The age range was 55 to 86 years (mean 74 years). Nineteen patients in each group were male and 36 were female. Statistical analysis was performed using Armitage's standardised normal deviate and the Fisher two-tailed exact probability test where appropriate.
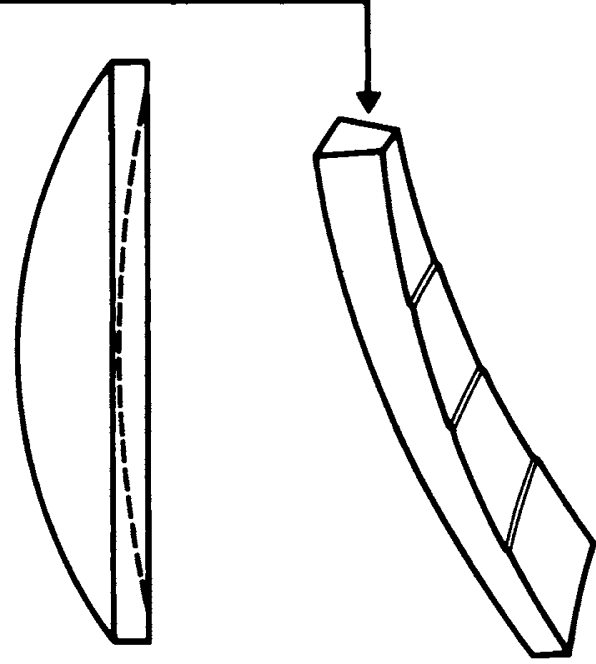

\section{Results}

Slit lamp examination revealed that 17 bifocal lenses $(31 \%)$ were decentered by more than $0.5 \mathrm{~mm}$ and three were decentered by more than $1.0 \mathrm{~mm}$, that is with reference to the centre of the pupil. The mean pupil size was $2.7 \mathrm{~mm}$ (range $2.0-4.0 \mathrm{~mm}$ ). Corrected distance acuity (Table I) was $6 / 9$ or better in all eyes save one bifocal eye which saw $6 / 12$ owing to mild senile macular degeneration. The number of eyes seeing 6/12 and N6 (which are comparable acuities) unaided was 39 in the bifocal group and 17 in the monofocal group $(\mathrm{p}<0.001)$.

\section{Reading ability}

Forty-six bifocal eyes (84\%) and 11 monofocal eyes $(20 \%)$ were able to read N8 or better with the distance correction (Table II). Twenty-nine $(53 \%)$ of bifocal eyes and one (2\%) of monofocal eyes were able to read N5 through the distance correction $(p<0.001)$.

The mean reading addition for a near point of $25 \mathrm{~cm}$ was $0.3 \mathrm{D}$ for the bifocal eyes and 2.2D for the monofocal eyes. Forty-four $(80 \%)$ of bifocal eyes compared with no monofocal eyes required a reading addition for $25 \mathrm{~cm}$ of less than 1.0D and 26 of the 50 eyes $(50 \%)$ with $3.5 \mathrm{D}$ bifocals required no addition at all. However, only 44 of the bifocal 
Table I Best corrected distance acuity

\begin{tabular}{lrrrc}
\hline & $6 / 5$ & $6 / 6$ & $6 / 9$ & $6 / 12$ \\
\hline 55 Bifocal eyes & 7 & 26 & 21 & 1 \\
55 Monofocal eyes & 19 & 25 & 11 & \\
\hline
\end{tabular}

Table II Reading ability of 55 matched eyes with the distance correction

\begin{tabular}{lcccccc}
\hline & & & & & & $\begin{array}{c}N 24 \\
\text { or } \\
\text { worse }\end{array}$ \\
\hline $\begin{array}{l}\text { Bifocal group } \\
\begin{array}{l}\text { Monofocal } \\
\text { group }\end{array}\end{array}$ & 29 & 11 & 6 & 9 & 0 & 0 \\
\hline
\end{tabular}

Table III Best corrected reading ability at between $25 \mathrm{~cm}$ and $35 \mathrm{~cm}$

\begin{tabular}{lccc}
\hline & $N 5$ & $N 6$ & $N 8$ \\
\hline Bifocal group & 44 & 6 & 5 \\
Monofocal group & 55 & 0 & 0 \\
\hline
\end{tabular}

group compared with all controls could be correctable to N5 (Table III) at a comfortable distance ( 35 to $25 \mathrm{~cm}$ ).

\section{Quality of vision}

Table IV gives the subjective response from patients to questions pertaining to the operated eye under question and concerning the quality of vision and presence of symptoms during daily activities. The eight patients with paired eyes had a separate questionnaire for each eye. The questions asked were:

1. Rate satisfaction with your vision in the eye recently operated on as good, fair or poor

2 . Rate the quality of your near vision in dim light as good, fair or poor

3. (a) Rate the quality of your distance vision in dim light as good, fair or poor

(b) Do you have problems in the dark? yes or no

4. Do you now manage most of the time without glasses?

5. Do you need to wear glasses for reading small print or equivalent?

6. With your operated eye and wearing glasses if you need them, do you notice

(a) glare or dazzle?

(b) haloes or rings in your vision?

(c) near vision blurring?

(d) near vision distortion?

(e) distance vision distortion or blurring?

(f) double for intermediate distance (e.g. the table)?

(g) a shorter range of vision for near than you would like?

7. Do you notice double vision with both eyes open? (positive answers from two patients with preoperative esotropia were excluded)

8. Do you have problems with lining things up (depth perception) e.g. pouring out tea, going down steps? (answers were only con-

Table IV Responses to Questionnaire

\begin{tabular}{|c|c|c|c|c|c|}
\hline & \multicolumn{2}{|c|}{$\begin{array}{c}\text { Monofocal } \\
\text { eyes }\end{array}$} & \multicolumn{2}{|c|}{$\begin{array}{l}\text { Bifocal } \\
\text { eyes }\end{array}$} & \multirow{2}{*}{$\begin{array}{c}\text { Statistical } \\
\text { significance } \\
p=\end{array}$} \\
\hline & $n$ & $\%$ & $n$ & $\%$ & \\
\hline 1. Satisfaction with operation rated good & 48 & 87 & 40 & 73 & ns \\
\hline 2. Poor near vision in dim light & 2 & 4 & 13 & 24 & 0.018 \\
\hline 3. Poor distance vision in dim light or the dark & 6 & 11 & 9 & 16 & ns \\
\hline 4. Manage without glasses & 33 & 60 & 38 & 69 & ns \\
\hline 5. Need reading glasses & 48 & 87 & 27 & 49 & $<0.001$ \\
\hline 6. (a) Glare or dazzle noticed & 21 & 38 & 21 & 38 & ns \\
\hline (b) Haloes or rings noticed & 3 & 5 & 8 & 15 & ns \\
\hline (c) Near vision blurring noticed & 6 & 11 & 28 & 51 & $<0.001$ \\
\hline (d) Near vision distortion noticed & 2 & 4 & 4 & 7 & ns \\
\hline (e) Distance blurring or distortion noticed & 10 & 18 & 9 & 16 & ns \\
\hline (f) Double at intermediate distance & 1 & 2 & 2 & 4 & ns \\
\hline (g) Reduced depth of near focus & 4 & 7 & 14 & 25 & 0.09 \\
\hline 7. Binocular diplopia noticed & 3 & 5 & 2 & 4 & ns \\
\hline 8. Difficulty with depth perception & 4 & 7 & 3 & 5 & ns \\
\hline
\end{tabular}




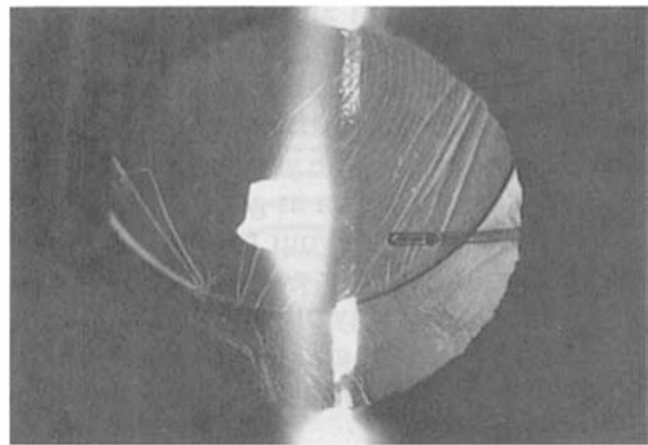

Fig. 2. Decentration of multizone bifocal lens (pupil dilated). VA $6 / 6$ and $N 5$ with $0.5 D$ reading addition.

sidered positive when the fellow eye was well sighted)

It will be seen that $69 \%$ of the bifocal group and $60 \%$ of the monofocal group stated that they could manage most of the time without glasses although $87 \%$ of the latter added that they needed reading glasses. In both groups the need for wearing glasses was sometimes coloured by the need to bring the fellow eye into use to maintain binocular vision. A difference in the quality of vision was revealed by the answers to questions $1,2,6(\mathrm{c})$ and $6(\mathrm{~g})$. Only $73 \%$ of the bifocal group rated their satisfaction with the operation as good. $24 \%$ of the bifocal group rated their near vision in dim light as poor. $51 \%$ noticed blurring for near compared with $11 \%$ in the control group $(\mathrm{p}<0.001)$. The visual symptom was a shadow superimposed over small print. $25 \%$ felt that focusing was difficult. An analysis of best corrected reading acuities (Table III) confirmed a reading deficit in $20 \%$ of the bifocal group.

The difference in distance acuity was less significant although a greater proportion of monofocal eyes could see $6 / 5$ (Table I). Of the eight patients in whom the fellow eye acted as control, six preferred the result from the bifocal lens. Two stated a preference for the monofocal because of a clearer and brighter visual image despite in each case an achievement with the bifocal of $6 / 12$ and N5 unaided.

Examination was made for ocular pathology to account for the reading deficit found objectively among the 11 eyes that could not be corrected to $\mathrm{N} 5$ at a distance between 35 and $25 \mathrm{~cm}$ (Table III): one patient had early Fuch's endothelial dystrophy to the same extent in each eye and despite early cataract could be corrected to a clear $6 / 9$ and $\mathrm{N} 5$ in the fellow eye. One patient had early senile macular degeneration but could still be corrected to 6/9 for distance. (Another patient outside this group also had early macular degeneration but was asymptomatic and had a best corrected acuity of $6 / 12$ and N5). No other defects could be detected. Visual acuities of patients in both groups were taken between four and eight months after surgery at a time when all posterior capsules were intact with no evidence of epithelial ingrowth or significant fibrosis. No fluorescein angiography was performed and although subclinical macular oedema could be ascribed to some patients there was no clinical evidence of this and no defects recorded on the Amsler grid.

There was no correlation of a reading deficit with age. Two of the eleven patients unable to read $\mathrm{N} 5$ were under the age of 66 years and two were over the age of 82 years. The mean age was 75 years.

There was a higher proportion of decentration among this small group with seven of eleven decentered more than $0.5 \mathrm{~mm}$ with reference to the centre of the pupil. However, this was not considered significant particularly as some with no symptoms had worse decentration (Fig. 2) and some with a reading deficit had normal centration (Fig. 3).

\section{Discussion}

It has long been known that some implanted

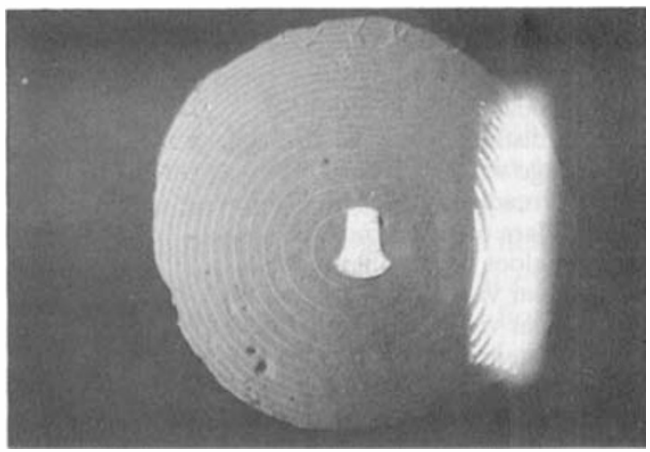

Fig. 3. Normal centration of bifocal lens. Best corrected $V A$ 6/9 and $N 8$. 
eyes are able to achieve good reading and distance vision without glasses. ${ }^{3}$ Apart from the higher resolving power of PMMA compared to the human lens, a second factor contributing to 'pseudo-accommodation' is that if the postoperative refraction is aimed at $-1.5 \mathrm{D}$ astigmatism, the depth of focus is increased. ${ }^{4}$ It is not surprising therefore that $20 \%$ of eyes with monofocal lenses were able to read $\mathrm{N} 8$ with the distance correction and that $31 \%$ were able to see $6 / 12$ and N6 without any aid.

It is against this relative success of modern lens implantation that the new generation of bifocal implants must be compared. This study leaves no doubt as to the effectiveness of the bifocal design with no less than $84 \%$ of eyes reading $\mathrm{N} 8$ or better with the distance correction and $71 \%$ seeing both $6 / 12$ and $\mathrm{N} 6$ unaided $(\mathrm{p}<0.001)$, but reservations exist over the quality of vision. When read at $35 \mathrm{~cm}$ N6 gives an acuity of $48 \%$ which is comparable with $6 / 12$ for distance. $6 / 9(66 \%)$ is comparable with $\mathrm{N} 4.5(64 \%)$ and so all eyes seeing $6 / 9$ for distance should be expected to read at least N5, particularly if additional powers are being allowed to bring the vision into focus as near as $25 \mathrm{~cm}$. The fact that $20 \%$ of eyes with bifocal lenses could not be corrected to $\mathrm{N} 5$ leads to the conclusion that for some, more than the $41 \%$ of light entering the eye needs to be in focus when reading small print.

In practical terms it was found that disappointment with the bifocal arose because some patients preferred to wear reading glasses when they may not be necessary in order to maintain binocularity. Others noted that their vision was 'shadowy' when attempting small print. Two of the eight patients in whom a monofocal lens in one eye could be compared directly with a bifocal in the other, declared that the bifocal vision was not as crisp as the monofocal vision and it may be that for some this is a price that has to be paid for the obvious advantage of lessening the dependence on reading glasses.

\section{References}

1 Futhey J: New IOL utilises diffractive optics. Ocular Surgery News 1988; Suppl to Sept 15, 1-4.

${ }^{2}$ Keats RH, Pearce JL, Schneider RT: Clinical results of the multifocal lens. J Cataract Refract Surg 1987; 13: 557-60.

${ }^{3}$ Percival SPB and Yousef KM: Treatment of uniocular aphakia. Br J Ophthalmol 1976; 60: 642-4.

${ }^{4}$ Huber C: Planned myopic astigmatism as a substitute for accommodation in pseudophakia. Am Intraocular Implant Soc J 1981; 7: 244-9. 